\title{
Monte Carlo simulation of scatter field for calculation of contrast of discs in synthetic CDMAM images
}

\author{
Oliver Díaz ${ }^{1}$, Mary Yip ${ }^{1}$, Jorge Cabello ${ }^{1 \star}$, David R. Dance ${ }^{2,3}$, Kenneth C. \\ Young $^{2,3}$, and Kevin Wells ${ }^{1}$ \\ 1 Centre for Vision, Speech and Signal Processing, Faculty of Engineering and \\ Physical Sciences, University of Surrey, Guildford, GU2 7XH, UK \\ o.diaz@surrey.ac.uk \\ 2 National Coordinating Centre for the Physics of Mammography, Royal Surrey \\ County Hospital, Guildford, GU2 7XX, UK \\ 3 Department of Physics, Faculty of Engineering and Physical Sciences, University of \\ Surrey, Guildford, GU2 7XH, UK
}

\begin{abstract}
This paper reports on a further development of an image simulation chain, and in particular, the inclusion of contrast degradation across an image using scatter to primary ratios calculated using Monte Carlo simulation. The Monte Carlo technique, using the Geant4 toolkit, has been implemented to model the scatter conditions when imaging the CDMAM phantom with commercial digital mammography. Observed differences between linear and cellular anti scatter grid are presented and discussed. These results support previous assumptions taken by Yip et al.[1].
\end{abstract}

Key words: Digital Mammography, Monte Carlo simulations, Scatter, anti-scatter grids, CDMAM phantom.

Presented at IWDM'2010 (Girona, Spain)

doi: 10.1007/978-3-642-13666-5_85

url: http://link. springer.com/chapter/10.1007\%2F978-3-642-13666-5_85

\section{Background}

New digital technologies are being developed for X-ray mammography which have the potential to improve cancer detection rates, but there is a need to investigate how these perform in comparison with conventional 2D digital mammography, and how these should be used in an optimal way. Since clinical trials to assess the performance of any mammography system are expensive, there is great interest in the development of computer-based models of the breast and imaging systems which can be used for evaluation and optimisation.

* Now at Institute for Corpuscular Physics (IFIC), University of Valencia/CSIC, Valencia, Spain. 
As a first step towards modelling patient images, and as a validation of the methodology, we are developing models for 2D imaging of the CDMAM phantom. This phantom is widely used for testing the performance of digital mammography systems in Europe. Previously, Yip et al[1] presented contrast detail curves calculated using a model incorporating heel effect, geometric and detector blurring and image noise. Loss of contrast due to scatter was assumed to be globally uniform across the image. In the present work, we address this previous assumption by incorporating scatter to primary ratio (SPR) maps calculated from Monte Carlo simulations.

\section{Method}

Figure 1a shows an adapted diagram from [1] of the simulation chain including the SPR component. The simulation chain has two parts. First, a noise free image is generated. Pixels values are assigned to a binary CDMAM template according to the exposure to be simulated and a heel effect mask is applied. Discs are added to the CDMAM template using the SPR calculated from the Monte Carlo simulations in order to calculate the contrast for each disc as explained below. The image is then blurred with an MTF filter. In the second part, a noise image is simulated using a Gaussian noise field and a noise filter calculated from the relevant Normalised Noise Power Spectrum(NNPS). This image is scaled according to an a-priori mean variance relationship for the detector. The final image is obtained after adding the blurred image and the scaled noise image.

Geometry. For the Monte Carlo simulations, the following components (from top to bottom) have been included in the setup according to their real dimensions and materials taken from manufacturer's specifications as illustrated in Figure 1b: (i) compression paddle, (ii) CDMAM phantom with a block of Perspex on top and bottom, (iii) breast support, (iv) anti-scatter grid with cover on top and bottom and (v) detector with its top cover as well. Selenium, cesium iodide and cesium bromide have been used for the Hologic, GE and Agfa detectors simulated in this work. The CDMAM test object has been simulated using a $3 \mathrm{~mm}$ thick Perspex layer and $0.5 \mathrm{~mm}$ thick Aluminium layer. $20 \mathrm{~mm}$ thick Perspex blocks were placed on top and bottom of CDMAM. The focal spot was considered as a point source $65 \mathrm{~cm}$ above the centre of chest wall edge of the detector. A top view diagram of the set up used for GE simulation is shown in Figure 2(a). The chest wall (CW) is located at position O. Outer area (1) represents the external dimensions of the system (detector, grid, breast support and compression paddle). (2) illustrates the position of the blocks of Perspex placed on top and bottom of CDMAM and (3) and (4) shows the location of the Perspex and Aluminium layers respectively used to simulate CDMAM.

Spectra. Table 1 describes the spectra used for the three systems simulated: Hologic Selenia, GE Essential and Siemens Mammomat 3000 using an Agfa computerised radiography plate. The energy spectrum has been simulated in 


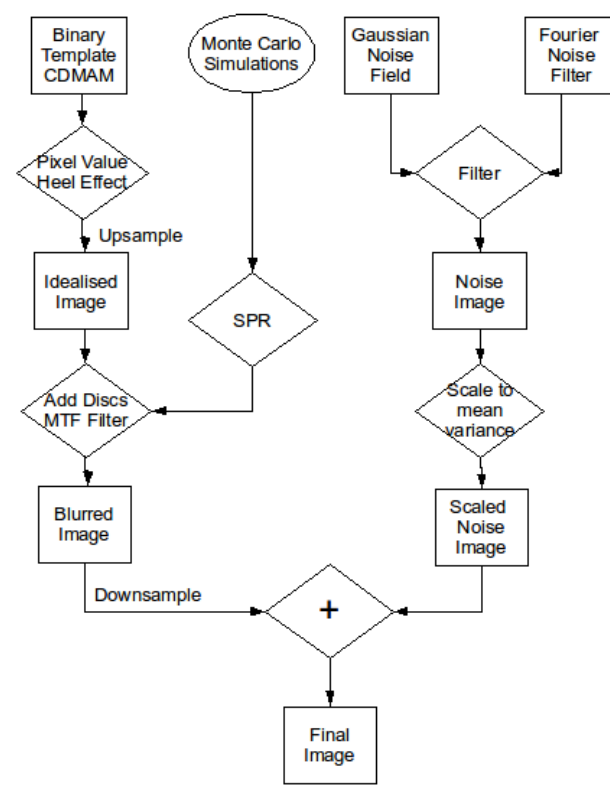

(a)

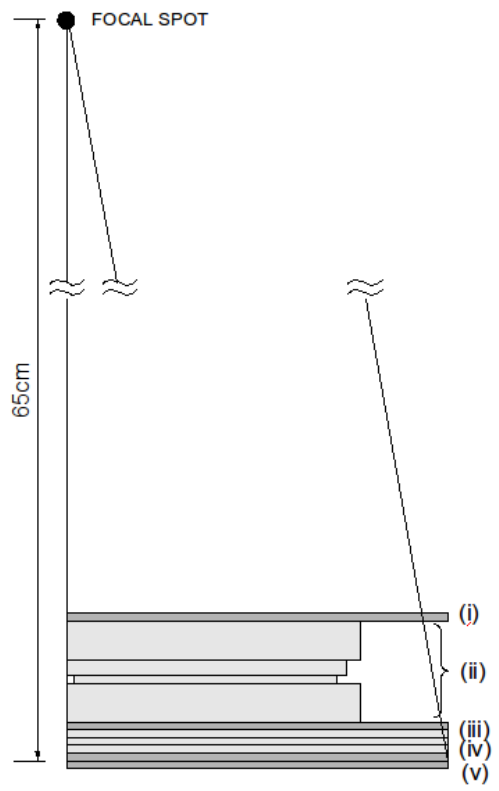

(b)

Fig. 1: (a) Diagram of Image Simulation Chain, adapted from [1], where the insertion of SPR map can be observed . (b) illustrates the setup used in Monte Carlo simulations with all the layers commented above. The numbered layers are discussed in the text.

Monte Carlo using data calculated from [2] and adjusted mathematically with a specific aluminium filter thickness to match the measured half value layer (HVL). Three simulations of $10^{10}$ X-ray photons have been simulated in Monte Carlo. These were summated to model each system.

Table 1: Spectra used in the simulations.

\begin{tabular}{ccc} 
System & Spectrum(Target/Filter) & HVL \\
\hline Hologic Selenia & W/Rh @ 30kVp & $0.45 \mathrm{~mm}$ \\
GE Essential & Rh/Rh @ 29kVp & $0.42 \mathrm{~mm}$ \\
Siemens/Agfa & Mo/Rh @ 28kVp & $0.41 \mathrm{~mm}$
\end{tabular}

Anti-scatter grid. Special attention has been paid to implementing the design of the moving focused anti-scatter grid to match the specifications of particular manufacturers systems. For the manufacturers chosen for this work, GE and Siemens/Agfa use linear grids, and a cellular grid is used by Hologic. In all the Monte Carlo simulations, the anti-scatter grid has been shifted several times 
along one axis (for the case of linear grids) or 2 axes (in the case of cellular grid) to cover a complete scatter grid unit cell(septa width + interspace width). The simulated anti-scatter grid was sufficiently large to cover the entire detector after every shift. The septa were positioned and angled so as to simulate a focused grid with the focal spot at a distance of $65 \mathrm{~cm}$ from the edge of the detector. Cover layers on the top and bottom of the grid have been included in the simulations.

Detector. The Monte Carlo simulation used the Geant 4 code $[3,4]$. Once a photon has left the X-ray source, it is tracked through all the layers above the detector until either it is completely absorbed by one of the layers (including the detector), or it leaves the system. During its passage through the geometry shown in Figure 1b, the photon is tagged as a scatter if it undergoes a Compton and/or Rayleigh interaction in any of the layers above the detector. When a photon reaches the detector, only Compton interactions and photoelectric absorption within the detector are taken into account in order to score the energy deposited. The energy deposited in the detector and its spatial location are stored according to the aforementioned flag (scattering, or primary if it does not interact with any layer above the detector).

SPR Calculation. The SPR was calculated using a similar method to that of [5], where the ratio of the image generated by energy deposited by scattered photons (affected by Compton or Rayleigh) within the detector over the image generated by energy deposited by primary photons gives the scatter to primary map(Equation 1). Here $I_{S c a}$ and $I_{P r i}$ represent the images generated by energy deposited inside the detector affected by scattering and primary photons respectively for one of the position of the grid. $I_{S c a, T}$ corresponds to the sum of all the $I_{S c a}$ images generated for each shifted grid position, while $I_{P r i, T}$ is similarly defined for $I_{P r i}$.

$$
\frac{S}{P}=\frac{\sum I_{S c a}}{\sum I_{P r i}}=\frac{I_{S c a, T}}{I_{P r i, T}}
$$

This expression is evaluated separately for each pixel. The SPR is generated using a detector comprising $1 \mathrm{~mm}^{2}$ pixels under the assumption that the scatter distribution does not contain fine structures. Then, using this assumption that the scatter distribution is a slowly varying function, the SPR image is median-filtered by an 11x11 kernel, providing a smoother, less statistically noisy representation of the SPR distribution. As the region of interest in this work is the CDMAM area, the image recorded in the detector is then cropped to the CDMAM's dimensions (inner area (4) shown in Figure 2a) giving a SPR image size of $152 \times 230$ pixels using the pixel dimension $\left(1 \mathrm{~mm}^{2}\right)$.

Contrast discs calculation using scatter map. Once the SPR map is generated and cropped to CDMAM's location, this is inserted in the image simulation chain to calculate the new contrast of the discs in the CDMAM as explained above. The contrast of each disc within the CDMAM is calculated using Equation 2, where $C_{o b s}$ represents the contrast of a disc observed at some particular 
location, $C_{p}$ is the relative contrast of this disc considering only primaries passing the CDMAM and blocks of Perspex on top and bottom with and without the gold disc of specific thickness and $\frac{S}{P}$ is the value of the SPR for the specific coordinates of the disc in the CDMAM image.

$$
C_{o b s}=\frac{C_{p}}{1+\frac{S}{P}}
$$

\section{Results}

Images of energy deposited by primary and scattered photons recorded in the GE detector after Monte Carlo simulations are shown in Figure 2b and 2c respectively. The resultant SPR image is presented also in Figure 2d. The mean value of SPR calculated in the CDMAM area is 0.170 with a standard deviation of 0.008 for this system. Figure 2a shows a top view of the GE system and block of Perspex and CDMAM dimensions are highlighted as mentioned above.

A 3D plot of the median-filtered SPR map shown in Figure $2 \mathrm{~d}$ is plotted in Figure 3a, where the the various edges of the components of the phantom are also shown. Figure 3b and 3c illustrate SPR values calculated by the mean of 10 profiles along planes parallel and perpendicular to the anode-cathode axis (profiles OA and BC of Figure 2a).

For the Siemens/Agfa system, the mean SPR value across the CDMAM location was 0.172 (standard deviation of 0.008 ) and for the Hologic system a mean SPR value of 0.06 (standard deviation of $8.22 \times 10^{-4}$ ) was found.

A study of the anti scatter grids used by GE (linear) and Hologic (cellular) was also undertaken. Figure 4 illustrates the equivalent profiles as seen above but in this case, SPR is plotted for both GE and Hologic system with and without anti scatter grid. Contrast improvement factors (CIF) of 1.50 and 1.60 was measured for GE and Hologic anti scatter grids respectively. Ratios of CIF between linear and cellular grids found by [6] varies from 0.91 to 0.95 for different thickness of breast phantoms for $30 \mathrm{kVp}$. In this work, the ratio of the CIF values of the GE and Hologic grids is 0.937 .

Automated scoring software for CDMAM using the CDCOM framework [7][8] has been used in this work for evaluation. A comparison of experimental acquired data, previous simulation data using a globally uniform SPR [1] and results from this work are shown in Figure 5 for GE systems and three different mean glandular doses(MGD).

\section{Discussion}

Considering Figure $3 \mathrm{~b}$, an increase of the SPR values is observed near the edge from area (1) to (2) and from (2) to (3)(4). Previously, [9] introduced this effect due to the compression paddle. A similar effect of increasing SPR at the edges is also described in [5]. Thus, components of the system such as the compression 


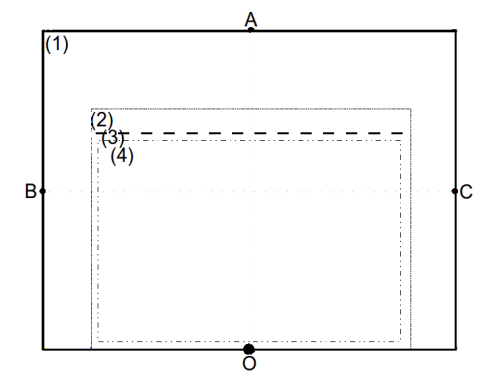

(a)

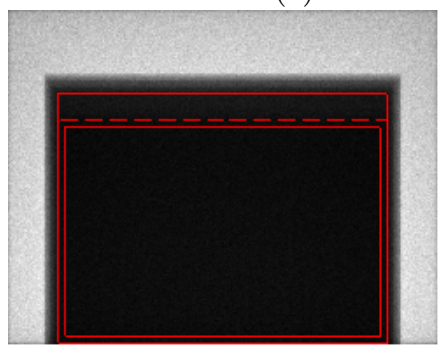

CW

(c)

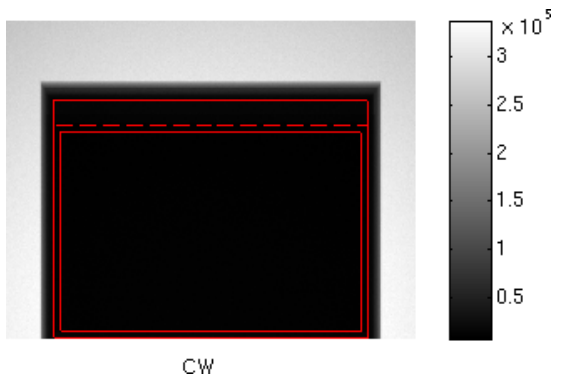

(b)
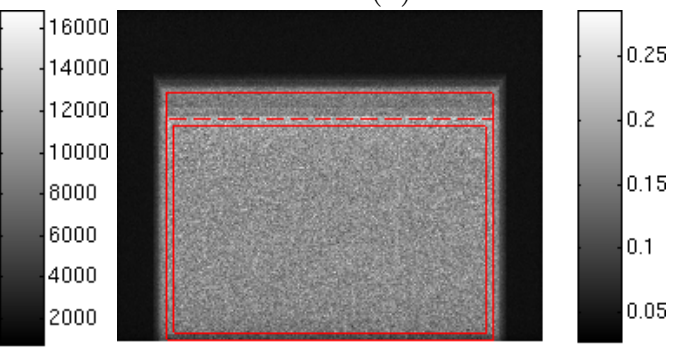

(d)

Fig. 2: Images generated from Monte Carlo Simulations: (a) top-view diagram of a GE system where outer area (1) represents system dimension, (2) block of Perspex on top and bottom of CDMAM and (3) and (4) Perspex and Aluminium layers of CDMAM respectively. (b) shows the energy deposited by primary photons in the detector $I_{P r i, T}$, (c) represents energy deposited in the detector due to scattered photons $I_{S c a, T}$ and (d) represents the SPR calculated using (b) and (c). Note that all layers shown in (a) are highlighted in (b),(c) and (d).

paddle or breast support may increase somewhat the scattering on the edge of phantoms and should be taking into account for scatter correction.

The cellular grid implemented here has better performance than the linear grid as expected. The very low scatter found with this set up when comparing with other cellular grid [6] can be attributed to wider septa and interspace. This also eliminates the increased scatter at the edges of CDMAM discussed previously.

It is observed in Figure 5 that images simulation using both uniform scatter and scatter calculated from Monte Carlo are within the error bars, so the results shown in this work support previous assumption used by [1]. Moreover, the simulation chain still have to be improved.

Acknowledgments. This work is part of the OPTIMAM project and is supported by CR-UK \& EPSRC Cancer Imaging Programme in Surrey, in association with the MRC and Department of Health (England). The author would 


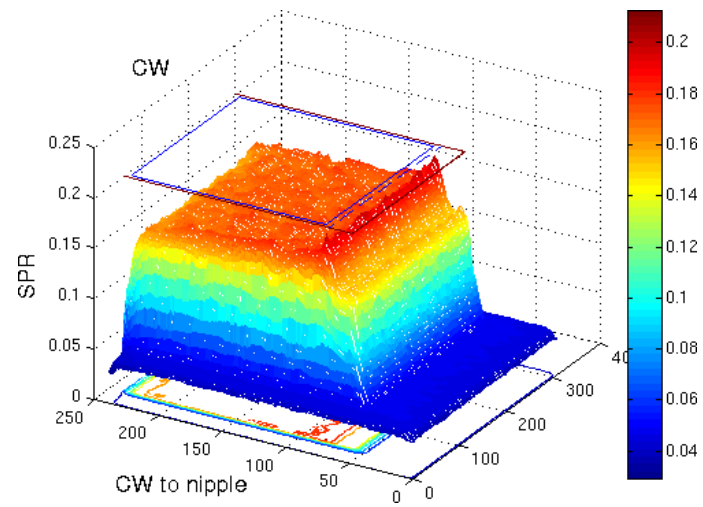

(a)

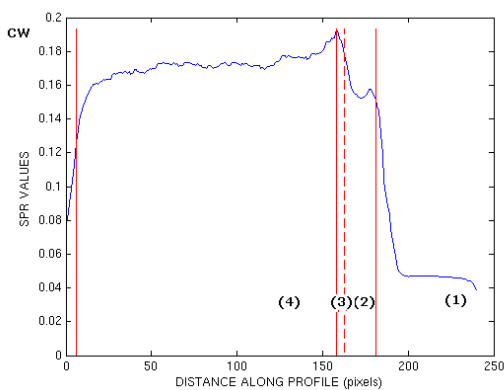

(b)

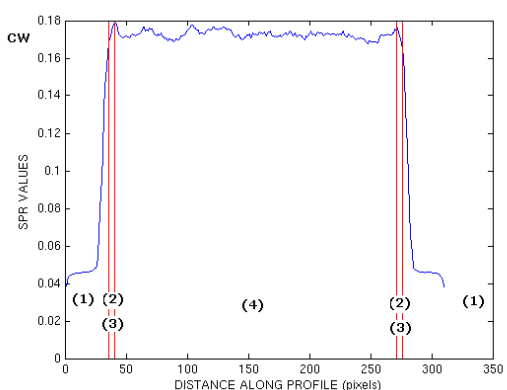

(c)

Fig. 3: (a) shows a 3D plot of the SPR map in the detector where CDMAM areas are highlighted. (b) and (c) represents the mean SPR values of 10 profiles along profile OA and BC illustrated in Figure 2a respectively. Vertical lines represents changes in dimension along the profiles $\mathrm{OA}$ and $\mathrm{BC}$ shown in Figure $2 \mathrm{a}$. Numerical labels (1-4) corresponds to regions shown in Fig. 2a.

like to thank GE, Hologic, Siemens and Agfa for providing technical information used in the simulations.

\section{References}

1. Yip, M., Alsager, A., Lewis, E., Wells, K., Young, K.C.:Validation of a digital mammography image simulation chain with automated scoring of CDMAM images. In: Krupinski, E.A.(Ed.) IWDM 2008.LNCS 5116, 409-416.Springer, Heidelberg,2008

2. Boone, J., Fewell, T., Jennings, J.: Molybdenum, rhodium and tungsten anode spectral models using interpolating polynomials with applications to mammography. Med. Phys., 24(2), 1863-1874, 1997.

3. Agostinelli, S. et al.: Geant4 - a simulation toolkit. Nuclear Instruments and Methods in Physics Research, 506(3), 250-303, 2003.

4. Allison, J. et al: Geant4 development and applications.IEEE Transactions on $\mathrm{Nu}-$ clear Science, 53(1), 270-278, 2006. 


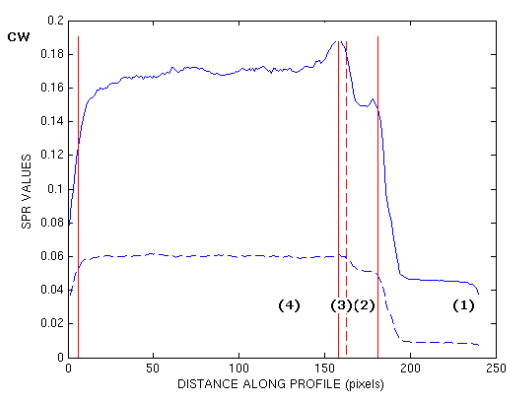

(a)

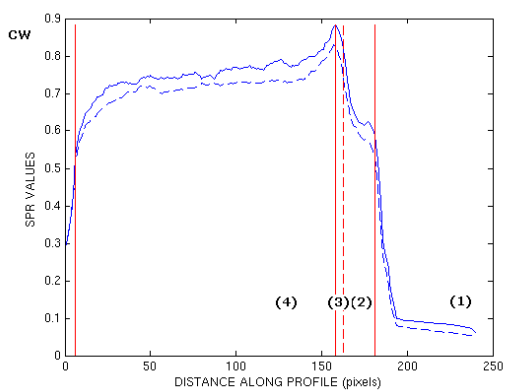

(b)

Fig. 4: Grid Study:In both figures, solid lines represent the SPR from the GE system and dashed lines from the Hologic system. (a) and (b) show the profiles $\mathrm{OA}$ with and without anti scatter grid. Vertical lines represents changes in dimension along the profiles $\mathrm{OA}$ and $\mathrm{BC}$ shown in Figure 2a. Numerical labels (1-4) corresponds to regions shown in Fig. 2a.

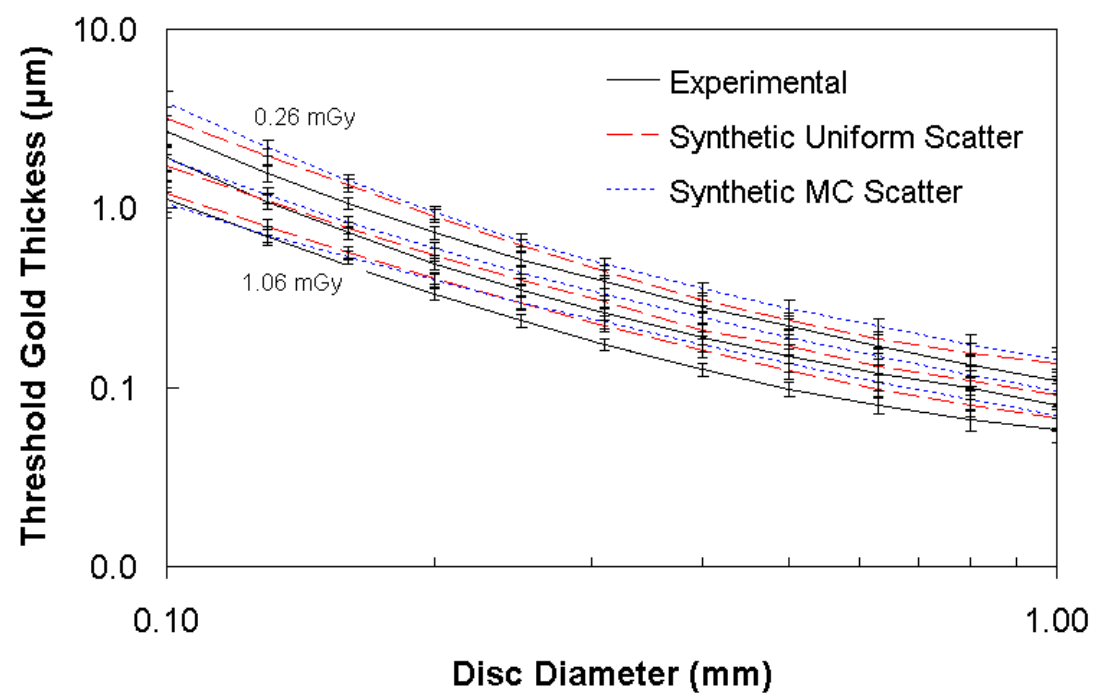

Fig. 5: Contrast detail curves for GE system. From top to bottom: 0.26, 0.52 and $1.06 \mathrm{mGy}$ MGD. Solid lines illustrate contrast detail measured from experimental data. Dashed and dotted lines represent contrast detail considering uniform scatter (from [1]) and calculating SPR from Monte Carlo simulations respectively. Errors bars indicate 2 standard errors of mean.

5. Sechopoulos, I., Suryanarayanan, S., Vedantham, S.,D’Orsi, C., Karellas, A.: Scatter radiation in digital tomosynthesis of the breast. Med. Phys., 34(2), 564-576, 2007. 
6. Rezentes, P.S., de Almeida, A., Barnes, G.T.: Mammography Grid Performance. Radiology, 210, 227-232, 1999.

7. Visser, M., Karssemeijer, N.: Manual CDCOM version 1.5: software for automated readout of CDMAM 3.4 images, www.euref .org

8. Young, K.C., Cook, J.J.H., Oduko, J.M.: Automated and human determination of threshold contrast for digital mammography systems. In: Astley, S.M., Brady, M., Rose, C., Zwiggelaar, R. (eds.) IWDM 2006. LNCS, vol. 4046, pp 266-272. Springer, Heidelberg (2006)

9. Dance, D.R. and Day, G.J.: The computation of scatter in mammography by Monte Carlo methods.Physics in Medicine and Biology, 29(3), 237-247, 1983. 